\title{
Evolução clínica e comportamento da função ventricular no pós-operatório tardio da cardiomioplastia
}

Luiz Felipe P. MOREIRA*, Noedir A. G. STOLF*, Edimar A. BOCCHI*, Fernando BACAL*, Paulo M. PÉGO-FERNANDES ${ }^{\star}$, Alfredo I. FIORELLI*, Henry ABENSUR ${ }^{\star}$, José C. MENEGHETTI*, Paulo S. GUTIERREZ*, Geraldo VERGINELLI*, Adib D. JATENE*

RBCCV 44205-253

MOREIRA, L. F. P.; STOLF, N. A. G.; BOCCHI, E. A.; BACAL, F.; PÊGO-FERNANDES, P. M.; FIORELLI, A. I.; ABENSUR, H.; MENEGHETTI, J. C.; GUTIERREZ, P.S.; VERGINELLI, G.; JATENE, A. D. - Evolução clínica e comportamento da função ventricular no pós-operatório tardio da cardiomioplastia. Rev. Bras. Cir. Cardiovasc., 10 (1): 3-17, 1995.

RESUMO: A cardiomioplastia tem sido proposta, como uma alternativa ao transplante cardíaco, no tratamento de pacientes com insuficiência miocárdica em fase avançada. O objetivo deste trabalho é estudar a evolução clínica e o comportamento da função ventricular no pós-operatório tardio desse procedimento em 34 pacientes portadores de cardiomiopatia dilatada, que foram operados no período de maio de 1988 a setembro de 1994. Vinte e sete pacientes estavam em classe funcional III e 7 pacientes em classe IV no préoperatório, apesar do uso de terapêutica clínica otimizada. A mortalidade hospitalar foi de 2,9\% e 1 paciente que evoluiu em choque cardiogênico foi submetido a transplante cardíaco 42 dias após a cardiomioplastia. 0 tempo de seguimento pós-operatório variou entre 2 e 73 meses, com média de 27,4 meses. Aos 6 meses de pós-operatório, 12 pacientes estavam em classe funcionall, 15 pacientes em classe ll e 3 pacientes em classe III $(p=0,001)$ em relação ao pré-operatório. Quatorze pacientes faleceram até 5 anos de pós-operatório e os indices de sobrevida foram $84,7 \%$ em 1 ano, $67,7 \%$ em 2 anos e $39,6 \%$ aos 5 anos de seguimento, sendo que, em 9 pacientes, os óbitos ocorreram por progressão da insuficiência cardiaca, e 5 pacientes faleceram subitamente. A análise de regressão de Gox mostrou que a mortalidade nos pacientes operados em classe funcional IV foi 5,5 vezes maior do que nos pacientes operados em classe III $(p=0,006)$, cuja sobrevida foi de $52,7 \%$ aos 5 anos de pós-operatório. O estudo sistemático da função ventricular através da angiografia com radioisótopos, da ecocardiografia com Doppler e do cateterismo cardíaco direito documentou a melhora da fraçăo de ejeçāo do ventrículo esquerdo (VE) de 19,8 3 para $23,9 \pm 7,2 \%(p<0,01)$, associada a modificaçōes significativas do encurtamento segmentar do VE, do volume e do índice sistólico, da pressão arterial média, das pressōes pulmonares e do índice de trabalho sistólico do V E, aos 6 meses de seguimento. No período pós-operatório tardio, a fração de ejeção e o encurtamento segmentar do $V E$ tenderam a cair e retornaram a valores semelhantes aos do pré-operatório em 5 anos, sendo observada no mesmo período a manutenção dos benefícios hemodinâmicos demonstrados após o procedimento. A eletrocardiografia dinâmica mostrou que a incidência de arritmias ventriculares e de fibrilação atrial năo se modificou após a cardiomioplastia. Finalmente, dos 5 pacientes que completaram 5 anos de seguimento, 3 faleceram por progressão de insuficiência cardiaca e 1 por morte súbita até os 73 meses de pós-operatório. Além disso, o estudo anatomopatológico mostrou a existência de infiltração gordurosa no enxerto muscular em 9 pacientes que faleceram entre 20 e 73 meses de pós-operatório, sendo observadas alteraçōes mais intensas do enxerto em pacientes com maior tempo de seguimento. Em conclusão, a cardiomioplastia melhora a condição clínica e a função ventricular esquerda de pacientes com cardiomiopatia dilatada. A sobrevida no pós-operatório tardio do procedimento é limitada, no entanto, em pacientes operados em classe funcional IV. Além disso, os benefícios da cardiomioplastia sobre a função sistólica do VE podem estar restritos a poucos anos de pósoperatório, fato que parece estar associado à degeneração tardia do enxerto muscular. cirurgia.

DESCRITORES: Cardiomioplastia. Insuficiência cardiaca, miocardiopatia, cirurgia. Miocardiopatias,

Trabalho realizado no Instituto do Coração do Hospital das Clínicas da Faculdade de Medicina da Universidade de São Paulo. São Paulo, SP, Brasil. Apresentado ao $22^{\circ}$ Congresso Nacional de Cirurgia Cardiaca. Recife, DF, 30 de março a 1 de abril, 1995.

* Do Instituto do Coração da Faculdade de Medicina da Universidade de Săo Paulo.

Endereço para correspondência: Luiz Felipe P. Moreira. Av. Dr. Enéas de Carvalho Aguiar, 44. Divisão Cirúrgica. CEP 05403-000 São Paulo, SP, Brasil. Tel. (011) 898.5432. Fax: (011) 282.2354 
MOREIRA, L. F. P.; STOLF, N. A. G.; BOCCHI, E. A.; BACAL, F.; PÊGO-FERNANDES, P. M.; FIORELLI, A. I.; ABENSUR, H.; MENEGHETTI, J. C.; GUTIERREZ, P. S.; VERGINELLI, G.; JATENE, A. D. - Evolução clínica e comportamento da função ventricular no pós-operatório tardio da cardiomioplastia. Rev. Bras. Cir. Cardiovasc., 10 (1): 3-17, 1995.

\section{INTRODUÇÃO}

A cardiomioplastia tem sido estudada por vários autores como uma alternativa cirúrgica no tratamento das miocardiopatias em fase avançada. Seus objetivos primários são a melhora do quadro de insuficiência cardiaca congestiva e a interrupção da progressão da doença de base em conseqüência do aumento da contratilidade e do reforço das paredes das câmaras ventriculares.

Resultados iniciais com o emprego da cardiomioplastia no tratamento de pacientes portadores de miocardiopatia de diversas etiologias têm demonstrado a melhora da fração de ejeção do ventrículo esquerdo (V E) e dos sintomas de insuficiência cardíaca após a realização do procedimento $12-16,18,29,32,34$. Alguns estudos sugerem, inclusive, que a cardiomioplastia pode melhorar a sobrevida de pacientes com grave comprometimento da função ventricular 10,33 .

Por outro lado, a mortalidade de pacientes submetidos a cardiomioplastia tem variado nas séries relatadas $8,12,16,19,29,30$ e documentação objetiva das modificações da função ventricular secundárias à realização do procedimento tem sido controversa $8,12,16,18,19,27$. Além disso, vários autores têm identificado limitações e fatores relacionados a um mau prognóstico com a utilização da cardiomioplastia, cuja importância é limitada pela sua associação a outros procedimentos e pela existência de afecções de diversas etiologias nas experiências analisadas $10,14,16$. A literatura atual não inclui ainda informações sobre a evolução clínica e o comportamento da função ventricular no seguimento tardio desse procedimento.

Neste trabalho, foram analisados a evolução clínica e o comportamento da função ventrícular e do ritmo cardíaco no pós-operatório tardio da cardiomioplastia, realizada como uma alternativa ao transplante cardíaco, em pacientes portadores de cardiomiopatia dilatada. A influência de parâmetros préoperatórios sobre os resultados tardios do procedimento foi também estudada.

\section{CASUÍSTICA E MÉTODOS}

\section{População Estudada}

Nesta investigação, foram estudados, prospectivamente, 34 pacientes consecutivos, portadores de cardiomiopatia dilatada, que foram submetidos a cardiomioplastia no Instituto do Coração do Hospital das Clínicas da Faculdade de Medicina da Universidade de São Paulo, no período de maio de 1988 a setembro de 1994.
Vinte e seis pacientes eram do sexo masculino e a idade variou entre 28 e 56 anos, com média de 45,2 anos. Todos os pacientes referiam sintomas de insuficiência cardíaca por um período superior a 1 ano antes da indicação do tratamento cirúrgico, tendo sido hospitalizados no mínimo uma vez nos últimos 6 meses por descompensação do quadro clínico. Vinte e sete pacientes estavam em classe funcional III e $7 \mathrm{em}$ classe fucional IV, segundo os critérios da "New York Heart Association" (NYHA), quando foi indicada a realização da cardiomioplastia. Todos os pacientes estavam utilizando doses otimizadas de digitálicos, diuréticos e inibidores da enzima de conversão. Cinco pacientes haviam utilizado drogas inotrópicas por via endovenosa no último mês que precedeu a operação.

Todos os pacientes apresentavam fração de ejeção de V E menor do que $30 \%$, documentada pela angiografia com radioisótopos, e hipertensão em território pulmonar (pressão média de capilar pulmonar maior do que $15 \mathrm{mmHg}$ ). $O$ cateterismo cardíaco demonstrou que nenhum dos pacientes apresentava obstruções significativas $(\geq 70 \%$ ) nas artérias coronárias e que 26 pacientes tinham insuficiência da valva mitral, que foi quantificada como moderada em 5 e leve em 21 pacientes. A biopsia endomiocárdica do ventrículo direito não mostrou a presença de processo inflamatório agudo no miocárdio em todos os pacientes.

Trinta pacientes estavam em ritmo sinusal e os outros 4 estavam em fibrilação atrial antes da operação. A eletrocardiografia dinâmica também mostrou que 13 pacientes apresentavam mais de 50 extra-sístoles ventriculares por hora e que episódios de taquicardia ventricular não sustentada foram observados em 16 pacientes. A avaliação da função pulmonar mostrou que 26 pacientes apresentavam insuficiência respiratória restritiva de grau discreto ou moderado. A maioria dos pacientes apresentava ainda contra-indicaçōes médicas ou psicossociais ao transplante cardíaco ou havia recusado a indicação do procedimento.

Nesta experiência, pacientes dependentes do uso de drogas inotrópicas endovenosas foram contra-indicados para a cardiomioplastia. Além disso, a insuficiência da valva mitral de grau importante, as arritmias refratárias à terapêutica clínica convencional, a cardiomegalia com diâmetro diastólico interno do VE maior do que $50 \mathrm{~mm} / \mathrm{m}^{2}$ de superfície corpórea e as doenças pulmonares ou sistêmicas irreversíveis foram também consideradas fatores de contra-indicação para o procedimento.

\section{Considerações Técnicas}

A cardiomioplastia foi realizada sem o uso de 
MOREIRA, L. F. P.; STOLF, N. A. G.; BOCCHI, E. A.; BACAL, F.; PÊGO-FERNANDES, P. M.; FIORELLI, A. I.; ABENSUR, H.; MENEGHETTI, J. C.; GUTIERREZ, P. S.; VERGINELLI, G.; JATENE, A. D. - Evolução clínica e comportamento da função ventricular no pós-operatório tardio da cardiomioplastia. Rev. Bras. Cir. Cardiovasc., 10 (1): 3-17, 1995.

circulação extracorpórea, através de duas vias de acesso diferentes: uma incisão lateral para a dissecção do músculo grande dorsal esquerdo e a esternotomia mediana para a fixação do enxerto muscular ao coração.

A dissecção e a transposição do músculo grande dorsal esquerdo para o interior do hemitórax esquerdo seguiram a técnica descrita por CARPENTIER \& CHACHQUES ${ }^{9}$. O enxerto muscular esquelético foi então disposto ao redor das câmaras ventriculares e fixado ao miocárdio através de suturas separadas, sendo realizado o envolvimento cárdio-costal posterior esquerdo. Dois eletrodos intramusculares (Medtronic SP-5528), implantados junto ao pedículo vasculonervoso do músculo grande dorsal, foram utilizados para estimular o enxerto muscular. O implante de um eletrodo epimiocárdico de sensibilidade e o implante do cardiomio-estimulador Medtronic SP-1005 encerraram o procedimento cirúrgico.

O procedimento anestésico inclui o uso sistemático de dopamina, dobutamina e vasodilatadores endovenosos, associados à manutenção de uma volemia adequada, com o objetivo de melhorar o comportamento hemodinâmico e de estabilizar a pressão arterial, inclusive durante as manobras de fixação do enxerto muscular ${ }^{1}$. O uso profilático de suporte inotrópico endovenoso foi mantido pelo menos durante a primeira semana de pós-operatório.

A estimulação elétrica do enxerto muscular foi iniciada 2 semanas após a operação, sendo seguido um protocolo progressivo de estimulação ${ }^{9}$. Os estímulos musculares começaram com pulsos isolados, sincronizados a cada dois batimentos cardiacos. O número de pulsos elétricos por contração muscular foi aumentado a cada 2 semanas, atingindo, após 2 meses de condicionamento, a freqüência de 30 Hertz. Cronicamente, a contração do enxerto muscular foi mantida sincronizada a todos os batimentos cardíacos ou a cada dois batimentos. A adequada sincronização entre a contração do músculo grande dorsal e a sístole ventricular foi obtida através de estudo ecocardiográfico, procurando-se fixar o início da estimulação muscular logo após o fechamento da valva mitral ${ }^{18}$.

\section{Protocolo de Seguimento Pós-Operatório}

Todos os pacientes foram seguidos pela mesma equipe médica e multiprofissional. A freqüência do acompanhamento ambulatorial foi quinzenal durante o período de adaptação do enxerto muscular, passando a ser trimestral no primeiro ano de seguimento e semestral a partir do segundo ano de pós-operatório. As avaliações da função ventricular e do comportamento do ritmo cardíaco foram realizadas em todos os pacientes antes da operação, aos 6 meses de pós-operatório e anualmente até o quinto ano de seguimento. As alterações da função dos ventrículos direito e esquerdo foram estudadas em repouso através da ventriculografia com radioisótopos, da ecocardiografia com Doppler e do cateterismo cardíaco direito. As características do ritmo cardíaco foram analisadas através da eletrocardiografia dinâmica.

A ventriculografia com radioisótopos foi obtida pela metodologia do equilíbrio, após a administração endovenosa de hemácias marcadas com technécio $99 \mathrm{~m}$. Foram adquiridas 32 imagens por ciclo cardíaco, num total de 10 milhões de contagens, com uma câmara Siemens modelo LEM+, sendo processadas em computador Microvax modelo 3300 . Foi utilizada a projeção oblíqua anterior esquerda com inclinação adequada para visibilizar os dois ventrículos e possibilitar o cálculo das frações de ejeção.

A avaliação pela ecocardiografia com Doppler foi realizada com equipamentos da marca Aloka, modelos SSD-730, SSD-860 e SSD-870. Foram utilizados transdutores setoriais, mecânicos ou eletrônicos, com freqüências de $2,0 \mathrm{MHz}$ a $3,5 \mathrm{MHz}$. Os parâmetros da função ventricular esquerda (diâmetros sistólico e diastólico de VE, encurtamento segmentar de VE) foram medidos através do modo $\mathrm{M}$ e da ecocardiografia bidimensional. $O$ volume sistólico do VE e o grau de insuficiência da valva mitral foram determinados pelo método Doppler.

O cateterismo do coração direito foi realizado com o paciente em decúbito dorsal horizontal, através da punção percutânea da veia jugular interna direita. Foi utilizado cateter de termodiluição do tipo Swan-Ganz (diâmetro 7F) para a medida das pressões, bem como para a determinação do débito cardíaco.

As características do ritmo cardíaco foram analisadas em computador da marca Marquette modelo $8000 \mathrm{~A}$. As gravações do eletrocardiograma foram obtidas em 3 derivações diferentes, durante um período mínimo de 22 horas. As variáveis eletrocardiográficas escolhidas foram: a freqüência cardíaca média, o ritmo cardíaco predominante, o número médio de extra-sístoles ventriculares por hora e o número de episódios de taquicardia ventricular não sustentada em 24 horas.

\section{Estudo Anatomopatológico}

Os pacientes que evoluíram para o óbito durante o seguimento pós-operatório foram, quando possível, submetidos a necropsia. Os exames foram 
MOREIRA, L. F. P.; STOLF, N. A. G.; BOCCHI, E. A.; BACAL, F.; PÊGO-FERNANDES, P. M.; FIORELLI, A. I.; ABENSUR, H.; MENEGHETTI, J. C.; GUTIERREZ, P. S.; VERGINELLI, G.; JATENE, A. D. - Evoluçāo clínica e comportamento da função ventricular no pós-operatório tardio da cardiomioplastia. Rev. Bras. Cir. Cardiovasc., 10 (1): 3-17, 1995.

realizados com a retirada dos órgãos em monobloco, seguindo-se o exame macroscópico completo e a análise histológica dos fragmentos de interesse para elucidação diagnóstica ou para investigação.

\section{Análise Estatística}

As curvas de sobrevida dos pacientes no pósoperatório tardio da cardiomioplastia foram calculadas pelo método de Kaplan-Meier. A diferença entre as curvas foi analisada pelo método de Logrank.

A influência de parâmetros clínicos e laboratoriais no pré-operatório sobre a sobrevida tardia dos pacientes foi avaliada através da análise isolada das variáveis, utilizando-se o teste $T$ de Student para as variáveis quantitativas e o teste exato de Ficher para as variáveis qualitativas. As variáveis consideradas significantes foram submetidas à análise de regressão de Cox. Foram excluídos dessa análise os pacientes com menos de um ano de seguimento, sendo considerados como óbitos os pacientes submetidos a transplante cardíaco de urgência.

Para as comparações das variáveis quantitativas estudadas entre o período pré-operatório e os diversos períodos de seguimento pós-operatório, foram realizados estudos através da análise de perfil, sendo utilizado o teste $F$ de Wilks. Para o estudo comparativo das variáveis qualitativas entre o período pré-operatório e os períodos de acompanhamento pós-operatório, foi utilizado o teste de McNemar. Nessas análises foram incluídas apenas as variáveis dos pacientes que apresentavam dados completos em relação aos períodos de seguimento estudados. Foram considerados significantes os valores com probabilidade inferior a 0,05.

\section{RESULTADOS}

\section{Período Pós-Operatório Imediato}

Houve um $(2,9 \%)$ óbito hospitalar nesta série, que ocorreu por infecção pulmonar e septicemia 9 dias após a operação. Um paciente que evoluiu com progressão da insuficiência cardíaca, em uso de drogas inotrópicas endovenosas e balão intra-aórtico, foi submetido a transplante cardiaco 42 dias após a cardiomioplastia.

Outros 2 pacientes apresentaram baixo débito cardíaco apesar do uso de drogas inotrópicas endovenosas, sendo também necessário o uso temporário de balão intra-aórtico. Complicaçōes pulmonares ocorreram em 3 pacientes e 1 paciente apre- sentou insuficiência renal $e$ isquemia mesentérica transitórias. Cinco pacientes apresentaram episódios de fibrilação ou flutter atrial no pós-operatório imediato, que foram revertidos em 4 pacientes com o uso de amiodarona endovenosa, enquanto que o outro paciente permaneceu cronicamente em fibrilação atrial.

O comprometimento isquêmico do enxerto muscular foi monitorizado através da variação da enzima creatinoquinase, que apresentou um valor médio de $946 \pm 582$ UI. Quatro pacientes apresentaram valores máximos da creatinoquinase acima de $1500 \mathrm{UI}$, nos quais não foi possível se detectar a contração do músculo grande dorsal no início do período de estimulação do enxerto, fato que não foi observado em nenhum dos outros pacientes.

\section{Seguimento Pós-Operatório Tardio}

O tempo de acompanhamento pós-operatório variou entre 2 e 73 meses, com média de 27,4 meses. Trinta e um pacientes completaram o protocolo de condicionamento do enxerto muscular e 1 paciente faleceu aos 2 meses de pós-operatório, devido a tromboembolismo pulmonar e choque cardiogênico. Durante o período de seguimento, não foram observadas complicações relacionadas aos eletrodos ou ao gerador de pulso. Nos primeiros 19 pacientes, o enxerto muscular foi estimulado cronicamente em sincronia com todos os batimentos cardíacos. Nos outros 12 pacientes, a contração do enxeto muscular foi mantida sincronizada a cada 2 batimentos cardíacos, na maior parte do período de seguimento.

Aos 6 meses de pós-operatório, 12 pacientes estavam em classe funcional I, 15 pacientes em classe funcional II e 3 pacientes em classe funcional III $(p=0,001)$ em relação ao pré-operatório, sendo observada situação semelhante do primeiro ao quarto ano de seguimento. O número de hospitalizações durante 0 período de pós-operatório também se modificou significativamente para 0,6 por pacienteano $(p=0,01)$.

Por outro lado, 13 dos 31 pacientes que completaram o período de adaptação do enxerto muscular faleceram até o quinto ano de pós-operatório; a causa do óbito estava relacionada a progressão da insuficiência cardíaca em 8 pacientes e 5 pacientes faleceram subitamente. Progressão de insuficiência cardiaca ocorreu em associação a complicações pulmonares em 4 pacientes e a insuficiência renal, acidente vascular cerebral, miocardite ou infecção de membro inferior nos outros 4 pacientes, respectivamente. Os indices de sobrevida, calculados pelo método de Kaplan-Meier, foram de $84,7 \%$ no primeiro ano, $69,7 \%$ aos 2 anos, $46,9 \%$ aos 3 
MOREIRA, L. F. P.; STOLF, N. A. G.; BOCCHI, E. A.; BACAL, F.; PÊGO-FERNANDES, P. M.; FIORELLI, A. I.; ABENSUR, H.; MENEGHETTI, J. C.; GUTIERREZ, P. S.; VERGINELLI, G.; JATENE, A. D. - Evolução clínica e comportamento da função ventricular no pós-operatório tardio da cardiomioplastia. Rev. Bras. Cir. Cardiovasc., 10 (1): 3-17, 1995.

GRÁFICO 1

CURVA DE SOBREVIDA (KAPLAN-MEIER) ATÉ 5 ANOS DE SEGUIMENTO APOS A CARDIOMIOPLASTIA. OS NÜMEROS ENTRE PARÊNTESES INDICAM OS PACIENTES QUE FORAM SEGUIDOS ATÉ O FINAL DE CADA PERIODO ANALISADO. AS PROBABILIDADES SÃO APRESENTADAS COM O LIMITE DE CONFIANÇA DE $70 \%$.

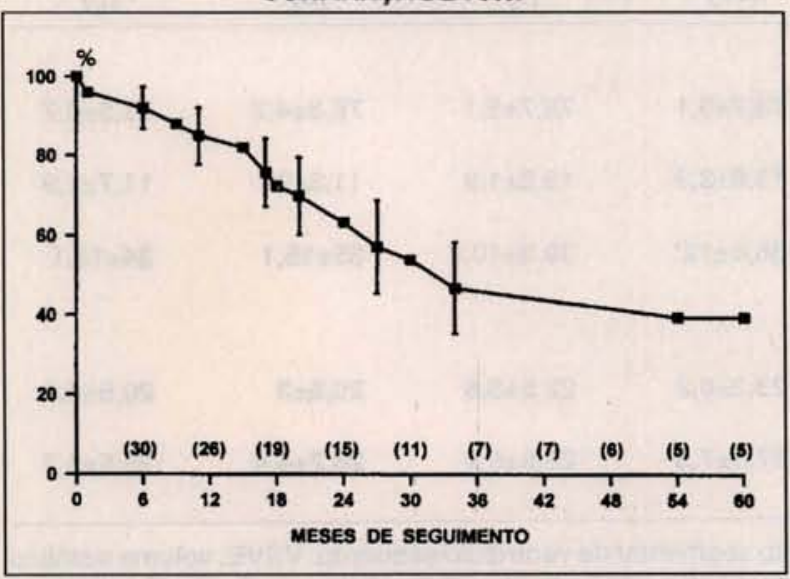

anos e $39,6 \%$ aos 5 anos de seguimento, como mostra o Gráfico 1.

A análise isolada das variáveis pré-operatórias relacionadas com o prognóstico identificou a existência de relação significativa entre a classe funcional e os valores da resistência vascular pulmonar no pré-operatório e os índices de sobrevida até os 5 anos de seguimento após a cardiomioplastia (Tabela 1). Quando esses parâmetros foram submetidos à análise de regressão de Cox, no entanto, apenas a classe funcional pré-operatória foi identificada como fator independente de prognóstico na evolução dos pacientes $(p=0,006)$. Essa análise mostrou ainda que os pacientes operados em classe funcional IV apresentaram um risco de mortalidade 5,5 vezes maior do que os pacientes operados em classe III

TABELA 1

ANÁLISE UNIFATORIAL DE FATORES ASSOCIADOS COM A SOBREVIDA

\begin{tabular}{lc}
\multicolumn{1}{c}{ PARÂMETRO } & SIGNIFICÂNCIA \\
\hline Classe funcional (NYHA) & $\mathrm{p}=0,005$ \\
Resistência vascular pulmonar & $\mathrm{p}=0,028$ \\
Pressão média de artéria pulmonar & $\mathrm{p}=0,096$ \\
Indice sistólico & $\mathrm{p}=0,144$ \\
Resistência vascular sistêmica & $\mathrm{p}=0,165$ \\
Índice cardiotorácico & $\mathrm{p}=0,168$ \\
Pressão média de átrio direito & $\mathrm{p}=0,179$ \\
Pressão média de capilar pulmonar & $\mathrm{p}=0,309$ \\
Pressão diastólica final de VE & $\mathrm{p}=0,358$ \\
Índice cardiaco & $\mathrm{p}=0,381$ \\
Diâmetro diastólico de VE & $\mathrm{p}=0,485$ \\
Fração de ejeção de VD & $\mathrm{p}=0,579$ \\
\hline
\end{tabular}

GRÁFICO 2

CURVAS DE SOBREVIDA (KAPLAN-MEIER) ATÉ 5 ANOS DE SEGUIMENTO APÓS A CARDIOMIOPLASTIA, CONFORME A CLASSE FUNCIONAL (C.F.) DOS PACIENTES NO PRÉ-OPERATÓRIO. AS PROBABILIDADES SÃO APRESENTADAS COM O LIMITE DE CONFIANCCA DE $70 \%$.

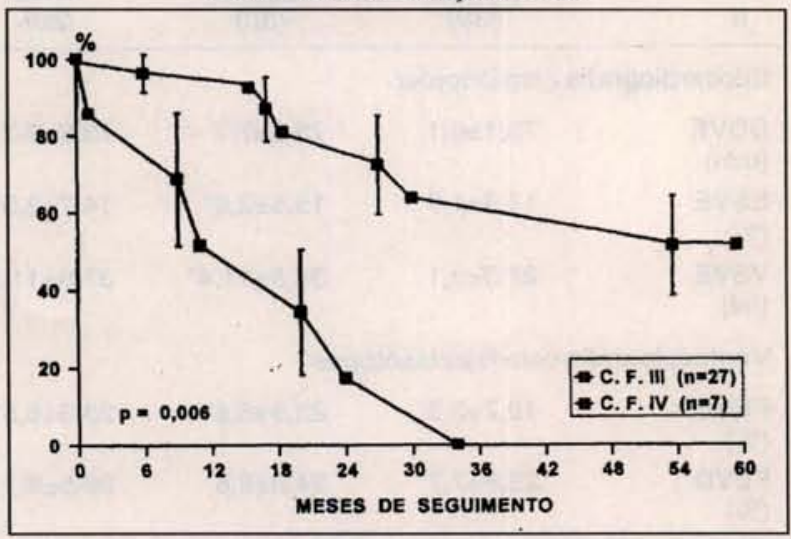

no período analisado (Gráfico 2). Os pacientes operados em classe funcional III apresentaram índices de sobrevida de $63,3 \%$ aos 3 anos e de $52,7 \%$ aos 5 anos de seguimento, enquanto que todos os pacientes em classe funcional IV faleceram até os 3 anos de pós-operatório.

Em relação à evolução clínica dos pacientes após 5 anos de seguimento, apenas um dos 5 pacientes que completaram aquele período permaneceu em classe funcional I até o sexto ano de pósoperatório. Três pacientes evoluíram depois do quinto ano em classe funcional III ou IV e faleceram em decorrência de progressão da insuficiência cardíaca aos 66, 67 e 73 meses de seguimento. O outro paciente, que também estava em classe funcional III, faleceu subitamente aos 62 meses de pós-operatório. Essas ocorrências foram responsáveis pela queda dos índices de sobrevida em pacientes submetidos à cardiomioplastia em classe funcional III para $21,1 \%$ aos 6 anos e para $10,5 \%$ aos 7 anos de seguimento.

\section{Comportamento da Função Ventricular}

A Tabela 2 mostra os valores da fração de ejeção dos ventrículos direito e esquerdo, obtidos através da angiografia com radioisótopos. A fração de ejeção de VE se elevou após a cardiomioplastia de $19,8 \pm 3$ para $23,9 \pm 7.2 \%$ ( $p=0,001)$. Na avaliação do comportamento tardio dessa variável, no entanto, foi documentado que os valores da fração de ejeção do VE se mantiveram significativamente elevados em relação ao pré-operatório apenas até o primeiro ano de pós-operatório, sendo observada a tendência de sua queda progressiva nos períodos 
MOREIRA, L. F. P.; STOLF, N. A. G.; BOCCHI, E. A.; BACAL, F.; PÊGO-FERNANDES, P. M.; FIORELLI, A. I.; ABENSUR, H.; MENEGHETTI, J. C.; GUTIERREZ, P. S.; VERGINELLI, G.; JATENE, A. D. - Evolução clínica e comportamento da funçāo ventricular no pós-operatório tardio da cardiomioplastia. Rev. Bras. Cir. Cardiovasc., 10 (1): 3-17, 1995.

TABELA 2

COMPORTAMENTO DOS PARÂMETROS NÄO INVASIVOS DA FUNÇÃO VENTRICULAR

\begin{tabular}{|c|c|c|c|c|c|c|c|c|}
\hline \multirow[b]{2}{*}{$n$} & & \multirow[b]{2}{*}{$\begin{array}{l}\text { Pré-Op. } \\
\quad(30)\end{array}$} & \multicolumn{6}{|c|}{ MESES DE SEGUIMENTO } \\
\hline & . & & $\begin{array}{l}6 \mathrm{~ms} \\
(30)\end{array}$ & $\begin{array}{c}12 \mathrm{~ms} \\
(26)\end{array}$ & $\begin{array}{c}24 m s \\
(15)\end{array}$ & $\begin{array}{l}36 \mathrm{~ms} \\
\text { (7) }\end{array}$ & $\begin{array}{l}48 m s \\
\text { (6) }\end{array}$ & $\begin{array}{l}60 \mathrm{~ms} \\
\text { (5) }\end{array}$ \\
\hline \multicolumn{9}{|c|}{ Ecocardiografia com Doppler } \\
\hline $\begin{array}{l}\text { DDVE } \\
(\mathrm{mm})\end{array}$ & & $73,1 \pm 6,1$ & $73,6 \pm 7,7$ & $73,8 \pm 7,7$ & $73,7 \pm 3,1$ & $75,7 \pm 5,1$ & $76,3 \pm 4,7$ & $77,5 \pm 6,7$ \\
\hline $\begin{array}{l}\text { ESVE } \\
(\%)\end{array}$ & & $11,9 \pm 1,9$ & $15,5 \pm 2,9^{*}$ & $14,7 \pm 3,5^{*}$ & $13,8 \pm 3,3$ & $13,2 \pm 1,9$ & $11,3 \pm 2$ & $11,7 \pm 1,9$ \\
\hline $\begin{array}{l}\text { VSVE } \\
(\mathrm{ml})\end{array}$ & & $27,3 \pm 9,1$ & $33,5 \pm 11,4^{*}$ & $37,8 \pm 11,8^{*}$ & $36,4 \pm 12^{*}$ & $39,3 \pm 10,8$ & $35 \pm 15,1$ & $34 \pm 13,1$ \\
\hline \multicolumn{9}{|c|}{ Ventriculografia com Radioisótopos } \\
\hline $\begin{array}{l}\text { FEVE } \\
(\%)\end{array}$ & & $19,7 \pm 3,3$ & $23,9 \pm 6,6^{*}$ & $23,3 \pm 6,5^{*}$ & $23,3 \pm 6,2$ & $22,5 \pm 3,6$ & $20,8 \pm 3$ & $20,5 \pm 3,6$ \\
\hline \multirow{2}{*}{\multicolumn{2}{|c|}{$\begin{array}{l}\text { FEVD } \\
(\%)\end{array}$}} & $25,8 \pm 7,7$ & $24,9 \pm 8,6$ & $26,5 \pm 8,1$ & $27,1 \pm 7,3$ & $26,5 \pm 5,4$ & $26,2 \pm 4,4$ & $25,5 \pm 5,7$ \\
\hline & & & & & 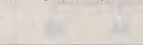 & 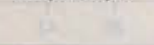 & 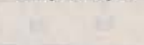 & 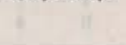 \\
\hline
\end{tabular}

DDEV: diâmetro diastólico de ventrículo esquerdo; ESVE: encurtamento segmentar de ventrículo esquerdo; VSVE: volume sistólico de ventrículo esquerdo; FEVE: fração de ejeção de ventrículo esquerdo; FEVD: fração de ejeção de ventrículo direito. Os números entre parêntesis indicam os pacientes avaliados em cada período. ${ }^{*}=p<0,05$ em relação aos valores pré-operatórios.

posteriores de seguimento, atingindo valores semeIhantes aos do pré-operatório aos 5 anos de pósoperatório (Gráfico 3).

Os dados obtidos pela ecocardiografia com Doppler também estão na Tabela 2. Aumentos significativos do encurtamento segmentar de VE e do volume sistólico de VE foram documentados aos 6 meses de pós-operatório. Por outro lado, a tendência a queda progressiva do encurtamento segmen-

GRÁFICO 3

CURVA DE TENDÊNCIA DO COMPORTAMENTO DA FRAÇÃO DE EJEÇÃO DO VENTRICCULO ESQUERDO ATÉ 5 ANOS DE SEGUIMENTO APÓS A CARDIOMIOPLASTIA. A LINHA MAIS FINA INDICA A TENDÊNCIA DO COMPORTAMENTO DA VARIÁVEL NOS 5 PACIENTES QUE COMPLETARAM O PERIODO DE SEGUIMENTOESTUDADO.

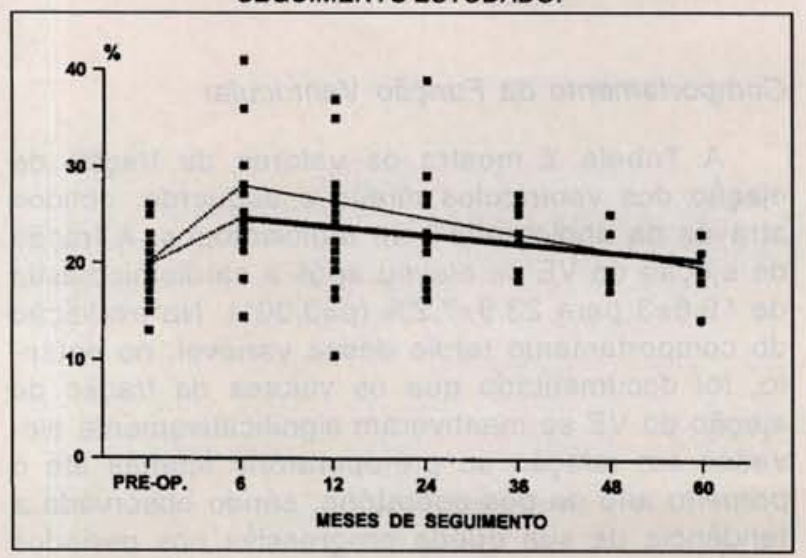

tar de VE após o primeiro ano de seguimento foi também documentada através desse método, enquanto que os valores do volume sistólico de $\mathrm{V} E$ mantiveram a melhora documentada aos 6 meses de pós-operatório durante todo o período estudado. A ecocardiografia com Doppler demonstrou ainda que não ocorreram modificações no grau de insuficiência da valva mitral durante todo o seguimento tardio.

\section{GRÁFICO 4}

CURVA DE TENDÊNCIA DO COMPORTAMENTO DA PRESSĀO MÉDIA DE CAPILAR PULMONAR EM 5 ANOS DE SEGUIMENTO APÓS A CARDIOMIOPLASTIA. A LINHA MAIS FINA INDICA A TENDÉNCIA DO COMPORTAMENTO DA VARIÁVEL NOS 5 PACIENTES QUE COMPLETARAM O PERIODO DE SEGUIMENTOESTUDADO.

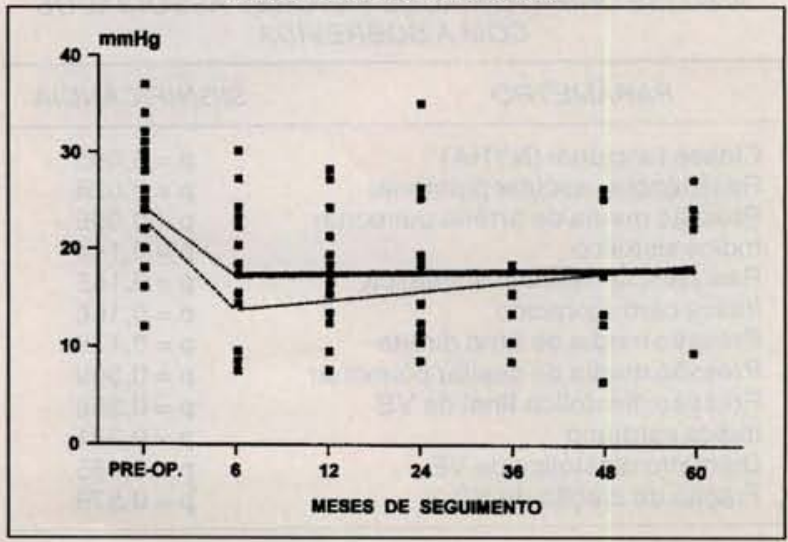


MOREIRA, L. F. P.; STOLF, N. A. G.; BOCCHI, E. A.; BACAL, F.; PÊGO-FERNANDES, P. M.; FIORELLI, A. I.; ABENSUR, H.; MENEGHETTI, J. C.; GUTIERREZ, P. S.; VERGINELLI, G.; JATENE, A. D. - Evolução clínica e comportamento da funçāo ventricular no pós-operatório tardio da cardiomioplastia. Rev. Bras. Cir. Cardiovasc., 10 (1): 3-17, 1995.

TABELA 3

COMPORTAMENTO DOS PARÂMETROS HEMODINÂMICOS

\begin{tabular}{|c|c|c|c|c|c|c|c|}
\hline \multirow[b]{2}{*}{$n$} & \multirow[b]{2}{*}{$\begin{array}{l}\text { Pré-Op. } \\
(30)\end{array}$} & \multicolumn{6}{|c|}{ Meses de Seguimento } \\
\hline & & $\begin{array}{l}6 \mathrm{~ms} \\
(30)\end{array}$ & $\begin{array}{c}12 \mathrm{~ms} \\
(26)\end{array}$ & $\begin{array}{c}24 \mathrm{~ms} \\
(15)\end{array}$ & $\begin{array}{c}36 \mathrm{~ms} \\
(7)\end{array}$ & $\begin{array}{c}48 \mathrm{~ms} \\
(6)\end{array}$ & $\begin{array}{c}60 \mathrm{~ms} \\
(5)\end{array}$ \\
\hline $\begin{array}{l}\text { PMAD } \\
(\mathrm{mmHg})\end{array}$ & $9 \pm 3,6$ & $9,1 \pm 5,3$ & $8,9 \pm 5,2$ & $9,1 \pm 6,3$ & $6,7 \pm 5,6$ & $7,2+5,6$ & $8+4,7$ \\
\hline $\begin{array}{l}\text { PMAP } \\
(\mathrm{mmHg})\end{array}$ & $36,1 \pm 11,2$ & $29,8 \pm 9,6^{*}$ & $30,3 \pm 9,4^{*}$ & $29,8 \pm 11,1^{*}$ & $24 \pm 6,8$ & $28,2+8,4$ & $30,5+7,5$ \\
\hline $\begin{array}{l}\text { PMCP } \\
(\mathrm{mmHg})\end{array}$ & $24,7 \pm 6,3$ & $18,5 \pm 6^{*}$ & $18,2 \pm 5,8^{*}$ & $17,9+7,7^{*}$ & $15,2 \pm 3,2^{*}$ & $15,5+6,1$ & $19+6,6$ \\
\hline $\begin{array}{l}\text { PAM } \\
(\mathrm{mmHg})\end{array}$ & $86,1 \pm 7,7$ & $93,1 \pm 10,6^{*}$ & $91,7 \pm 11,1$ & $93,2+8,8^{*}$ & $96,2 \pm 12,4^{*}$ & $98,8+15,3$ & $90,3+7,9$ \\
\hline $\begin{array}{l}\text { IC } \\
\left(\mathrm{I} / \mathrm{min} \cdot \mathrm{m}^{2}\right)\end{array}$ & $1,94 \pm 0,31$ & $2,02 \pm 0,4$ & $2,04 \pm 0,44$ & $1,97 \pm 0,26$ & $2,3 \pm 0,3$ & $2,17+0,26$ & $2,18+0,2$ \\
\hline $\begin{array}{l}\text { IS } \\
\left(\mathrm{ml} / \mathrm{m}^{2}\right)\end{array}$ & $20,6 \pm 3,1$ & $24 \pm 5,3^{*}$ & $24,7 \pm 6,8^{\star}$ & $25 \pm 6,7^{\star}$ & $28,9 \pm 4,4^{*}$ & $27+6,8$ & $25,7+4,9$ \\
\hline $\begin{array}{l}\text { ITSVD } \\
\text { (g.m/m²) }\end{array}$ & $7,4 \pm 2,1$ & $6,9 \pm 2,8$ & $7 \pm 3,6$ & $6,9 \pm 2,6$ & $6,7 \pm 1,4$ & $7,3+0,7$ & $7,7+0,7$ \\
\hline $\begin{array}{l}\text { ITSVE } \\
\left(\mathrm{g} \cdot \mathrm{m} / \mathrm{m}^{2}\right)\end{array}$ & $17,5 \pm 5,2$ & $25,5 \pm 9,1^{*}$ & $25,6 \pm 9,4^{\star}$ & $25,4 \pm 7^{*}$ & $30,9 \pm 9,5^{\star}$ & $29,7+10,9^{*}$ & $25,5+8,5$ \\
\hline $\begin{array}{l}\text { RVP } \\
\text { (dina.s.cm-5) }\end{array}$ & $288 \pm 166$ & $271 \pm 96$ & $282 \pm 136$ & $267 \pm 94$ & $183 \pm 107$ & $288+165$ & $236+70$ \\
\hline $\begin{array}{l}\text { RVS } \\
\text { (dina.s.cm-5) }\end{array}$ & $1862 \pm 258$ & $1962 \pm 329$ & $1882 \pm 393$ & $1930 \pm 365$ & $1748 \pm 166$ & $1838+305$ & $1638+141$ \\
\hline
\end{tabular}

PMAD: pressão média de átrio direito; PMAP: pressão média de artéria pulmonar; PMCP: pressão média de capilar pulmonar; PAM: pressão arterial média; IC: índice cardíaco; IS: índice sistólico; ITSVD: índice de trabalho sistólico de ventrículo direito; ITSVE: índice de trabalho sistólico de ventrículo esquerdo; RVP: resistência vascular pulmonar; RVS: persistência vascular sistêmica. Os números entre parêntesis indicam os pacientes avaliados em cada período. ${ }^{*}=p<0,05$ em relação aos valores pré-operatórios.

A avaliação do comportamento hemodinâmico mostrou que aumentos significativos da pressão arterial, do índice sistólico e do índice de trabalho sistólico de VE foram associados com quedas

\section{GRÁFICO 5}

CURVA DE TENDÊNCIA DO COMPORTAMENTO DO ÍNDICE DE TRABALHO SISTÓLICO DO VENTRICCULO ESQUERDO EM 5 ANOS DE SEGUIMENTO APÓS A CARDIOMIOPLASTIA. A LINHA MAIS FINA INDICA A TENDENNCIA DO COMPORTAMENTO DA VARIIÁVEL NOS 5 PACIENTES QUE COMPLETARAM O PERIODO DE SEGUIMENTO ESTUDADO.

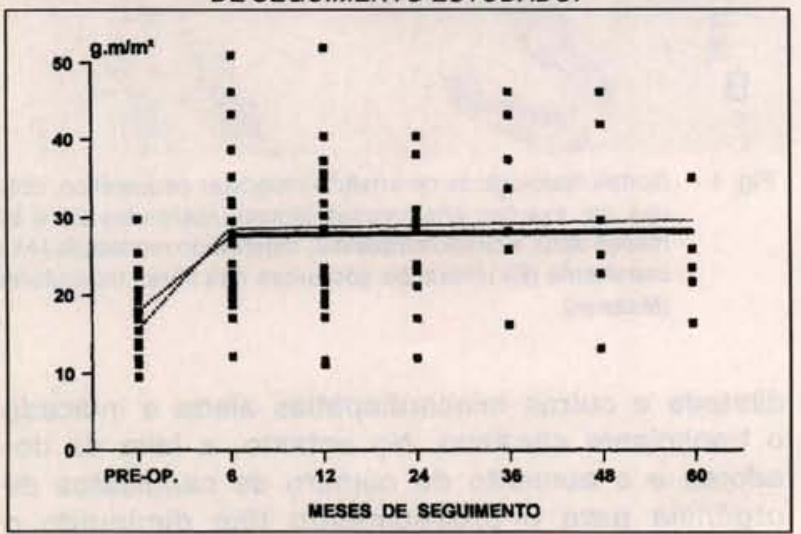

significativas da pressão média de artéria pulmonar e da pressão média de capilar pulmonar aos 6 meses de pós-operatório (Tabela 3 ). Durante o seguimento tardio, esse estudo documentou a tendência a manutenção dos benefícios hemodinâmicos da cardiomioplastia por até 5 anos de seguimento. $O$ Gráfico 4 mostra a curva de comportamento da pressão média de capilar pulmonar, enquanto que o comportamento do índice de trabalho sistólico de VE é apresentado no Gráfico 5.

\section{Comportamento do Ritmo Cardíaco}

A Tabela 4 mostra que a avaliação do comportamento da freqüência cardíaca média e da incidência de extra-sístoles ventriculares e de episódios de taquicardia ventricular não sustentada pela eletrocardiografia dinâmica não mostrou modificações significativas desses parâmetros durante todo o período de pós-operatório analisado, fato também observado a respeito da incidência de fibrilação atrial.

\section{Estudo Anatomopatológico}

Doze dos 18 pacientes que faleceram durante 
MOREIRA, L. F. P.; STOLF, N. A. G.; BOCCHI, E. A.; BACAL, F.; PÊGO-FERNANDES, P. M.; FIORELLI, A. I.; ABENSUR, H.; MENEGHETTI, J. C.; GUTIERREZ, P. S.; VERGINELLI, G.; JATENE, A. D. - Evolução clínica e comportamento da função ventricular no pós-operatório tardio da cardiomioplastia. Rev. Bras. Cir. Cardiovasc., 10 (1): 3-17, 1995.

TABELA 4

COMPORTAMENTO DAS VARIÁVEIS ELETROCARDIOGRÁFICAS

\begin{tabular}{|c|c|c|c|c|c|c|c|}
\hline \multirow[b]{2}{*}{ (n) } & \multirow[b]{2}{*}{$\begin{array}{l}\text { Pré-Op. } \\
(30)\end{array}$} & \multicolumn{5}{|c|}{ Meses de Seguimento } & \multirow[b]{2}{*}{$\begin{array}{c}60 \mathrm{~ms} \\
(5)\end{array}$} \\
\hline & & $\begin{array}{l}6 \mathrm{~ms} \\
(30)\end{array}$ & $\begin{array}{c}12 \mathrm{~ms} \\
(26)\end{array}$ & $\begin{array}{c}24 \mathrm{~ms} \\
(15)\end{array}$ & $\begin{array}{l}36 \mathrm{~ms} \\
(7)\end{array}$ & $\begin{array}{l}48 \mathrm{~ms} \\
(6)\end{array}$ & \\
\hline $\begin{array}{l}\mathrm{FC} \\
\text { (bat/min) }\end{array}$ & $89,1+14$ & $84,9+14,3$ & $82,9+15,5$ & $82,7+11,7$ & $86,7+10,6$ & $87,2+12$ & $84,6+11,5$ \\
\hline $\begin{array}{l}\text { FA } \\
\text { (n. pac.) }\end{array}$ & $4(13,3 \%)$ & $5(16,6 \%)$ & $5(19,2 \%)$ & $4(26,6 \%)$ & $1(14,2 \%)$ & $1(16,6 \%)$ & $1(20 \%)$ \\
\hline $\begin{array}{l}\mathrm{ESV} \\
(\mathrm{n} / \mathrm{h})\end{array}$ & $97+203$ & $71+165$ & $54+88$ & $55+108$ & $77+78$ & $51+37$ & $40+52$ \\
\hline $\begin{array}{l}\text { TVNS } \\
\text { (epis/24hs) }\end{array}$ & $3,1+6,9$ & $1,5+2,7$ & $0,5+1,7$ & $0,7+1,2$ & $1,4+2,7$ & $0,3+0,5$ & $0,8+1,2$ \\
\hline
\end{tabular}

FC: freqũência cardiaca média em 24 horas; FA: fibrilação atrial; ESV: extra-sístoles ventriculares; TVNS: taquicardia ventricular não sustentada. Os números entre parêntesis indicam os pacientes avaliados em cada período.

o seguimento pós-operatório foram submetidos à necropsia. Três pacientes faleceram durante o primeiro ano de seguimento, 3 entre 1 e 2 anos, 2 entre 2 e 5 anos e 4 após os 5 anos de seguimento. As causas clínicas dos óbitos foram: progressão da insuficiência cardíaca em 9 pacientes, tromboembolismo pulmonar em 1 paciente e morte súbita em 2 pacientes. Em 11 desses pacientes, o enxerto muscular foi estimulado cronicamente em sincronia com todos os batimentos cardíacos e apenas 1 paciente que faleceu aos 18 meses de seguimento foi mantido com a estimulação muscular sincronizada a cada 2 batimentos cardíacos.

O estudo anatomopatológico do enxerto muscular revelou a presença de fibrose perimisial e atrofia severa das fibras em 1 paciente que faleceu aos 10 meses de seguimento e havia apresentado elevação importante dos níveis plásmaticos da enzima creatinoquinase no pós-operatório imediato. Nove pacientes apresentavam sinais de infiltração de tecido gorduroso, que foi quantificada como leve em 2 casos, como moderada em 3 , como importante em 2 e como severa em 2 (Figura 1). O Gráfico 6 mostra que o grau de infiltração gordurosa nos enxertos musculares esqueléticos submetidos a estimulação elétrica prolongada foi influenciado significativamente pelo tempo de seguimento pós-operatório.

\section{COMENTÁRIOS}

A melhor compreensão da fisiopatologia da insuficiência cardiaca 36 trouxe novos avanços no campo da terapêutica medicamentosa, cujos resultados levaram ao aumento significativo da sobrevida de pacientes com insuficiência miocárdica em fase avançada ${ }^{37}$. Apesar desse fato, em um grande número de pacientes portadores de cardiomiopatia

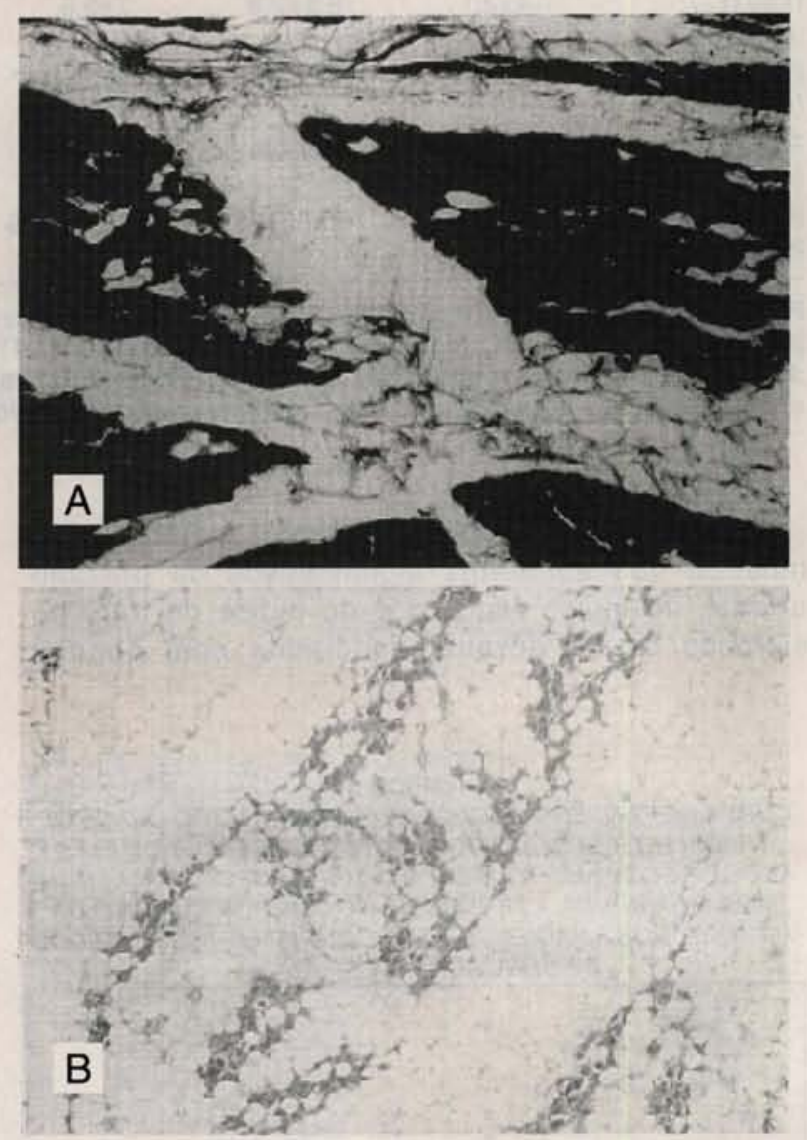

Fig. 1 - Cortes histológicos do enxerto muscular esquelético, obtidos em exames anatomopatológicos realizados 24 e 67 meses após a cardiomioplastia, mostrando moderada (A) e importante (B) infiltraçāo gordurosa das fibras musculares (Masson).

dilatada e outras miocardiopatias ainda é indicado o transplante cardíaco. No entanto, a falta de doadores e o aumento do número de candidatos de urgência para o procedimento têm diminuído o 
MOREIRA, L. F. P.; STOLF, N. A. G.; BOCCHI, E. A.; BACAL, F.; PÊGO-FERNANDES, P. M.; FIORELLI, A. I.; ABENSUR, H.; MENEGHETTI, J. C.; GUTIERREZ, P. S.; VERGINELLI, G.; JATENE, A. D. - Evolução clínica e comportamento da função ventricular no pós-operatório tardio da cardiomioplastia. Rev. Bras. Cir. Cardiovasc., 10 (1): 3-17, 1995.

GRÁFICO 6

RELAÇĀO ENTRE O GRAU DE INFILTRAÇĀO GORDUROSA DOS ENXERTOS MUSCULARES NOS ESTUDOS

ANATOMOPATOLOGICOS EO TEMPO DE SEGUIMENTO APÓS A CARDIOMIOPLASTIA. NESSES ESTUDOS A DEGENERAÇÃO GORDUROSA FOI QUANTIFICADA COMO LEVE (1), MODERADA (2), IMPORTANTE (3) OU GRAVE (4).

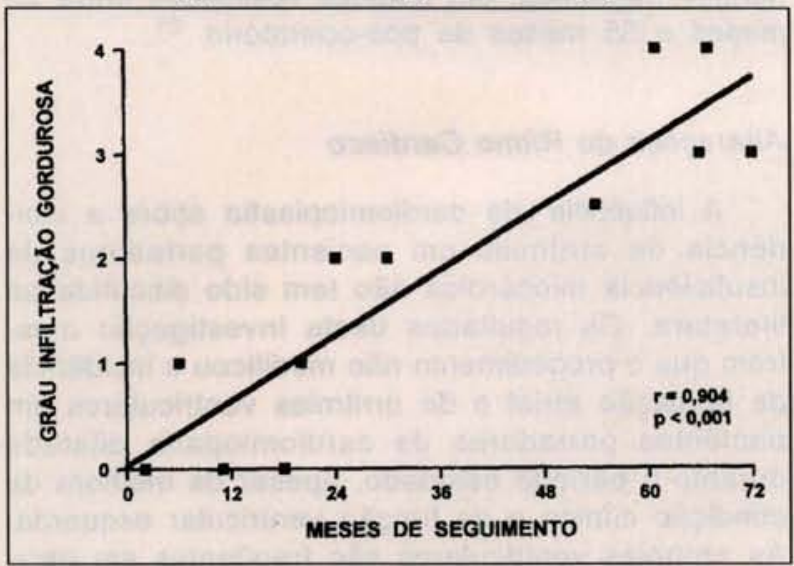

número de transplantes realizados em pacientes ambulatoriais $^{39}$, justificando a necessidade de outras alternativas cirúrgicas de tratamento para os pacientes que não conseguem manter uma boa qualidade de vida e um bom prognóstico apenas com a terapêutica medicamentosa. Essa população representa atualmente mais de 2.200 pacientes nas filas de espera para o transplante cardíaco só nos Estados Unidos, com menos de $5 \%$ de probabilidade de receber um coração a cada mês ${ }^{39}$.

Nesta investigação, a cardiomioplastia foi empregada, como uma alternativa ao transplante cardíaco, no tratamento de pacientes portadores de cardiomiopatia dilatada. A mortalidade hospitalar foi de apenas $2,9 \%$ e foi documentada a melhora significativa do quadro clínico e da função ventricular esquerda dos pacientes nos primeiros anos de pósoperatório. Por outro lado, a sobrevida tardia após a cardiomioplastia foi influenciada pela gravidade da condição clínica dos pacientes no pré-operatório, sugerindo que a indicação desse procedimento deva ser limitada a fases mais precoces de comprometimento da função ventricular. Além disso, embora o comportamento da função ventricular até o quinto ano de pós-operatório tenha sido caracterizado pela manutenção dos benefícios hemodinâmicos observados após o procedimento, foi também observada a queda progressiva da fração de ejeção e do encurtamento segmentar do VE, que atingiram níveis semelhantes aos do pré-operatório ao final do período analisado. Esse fato foi seguido pela má evolução clínica dos pacientes após 5 anos de seguimento, estando provavelmente relacionado à ocorrência de infiltração gordurosa nos enxertos musculares submetidos a estimulação elétrica prolongada.

\section{Alterações da Função Ventricular}

Vários autores têm demonstrado a elevação significativa da fração de ejeção do VE após a realização da cardiomioplastia entre $20 \%$ e $30 \%$ em relação aos valores pré-operatórios, através de métodos não invasivos $10,14,27,29,30$. Embora esse fato também tenha sido observado neste estudo, aumentos mais expressivos desse parâmetro foram previamente documentados através da cineventriculografia esquerda em 10 pacientes desta série ${ }^{6} \mathrm{e}$ em outros relatos da literatura 10, 19. A análise computadorizada da movimentação regional das paredes do VE pela cineventriculografia revela, adicionalmente, que a elevação pós-operatória da fração de ejeção está relacionada à melhora global da contratilidade e modificações na forma da câmara ventricular 6 .

Embora a melhora da função sistólica do ventrículo esquerdo seja responsável pela melhora significativa dos parâmetros hemodinâmicos em estudos experimentais $22,26,31$, o fato não tem sido observado clinicamente. Nesta investigação, no entanto, aumentos significativos do índice sistólico e do índice de trabalho sistólico do VE foram documentados após o período de adaptação do enxerto muscular, na presença de diminuição significativa das pressões em território pulmonar. A indicação da cardiomioplastia, nesta série, apenas em pacientes com comprometimento importante do débito cardíaco e hipertensão pulmonar, ao contrário do observado em outras experiências $10,19,29$, pode explicar os resultados obtidos. Entretanto, esses resultados podem ter sido também influenciados pela manutenção do enxerto muscular sincronizado a todos os batimentos cardíacos na maioria dos casos estudados.

Por outro lado, apesar da melhora dos parâmetros relatados, não ocorreram modificaçōes significativas do índice cardíaco e das resistências vasculares em repouso durante o período desta investigação. Além disso, a pressão arterial média manteve-se mais elevada do que no pré-operatório durante todo o período de seguimento, situação que pode ser explicada pela manutenção pós-operatória de alterações periféricas decorrentes do aumento da atividade neuro-hormonal nesses pacientes ${ }^{4}$. Apesar desse fato, um estudo realizado durante teste de esforço em esteira ergométrica demonstrou a elevação significativa do índice cardíaco no pós-operatório da cardiomioplastia, associada à queda das pressões em território pulmonar, no pico máximo do exercício, indicando a melhora da reserva contrátil do miocárdio 5 . 
MOREIRA, L. F. P.; STOLF, N. A. G.; BOCCHI, E. A.; BACAL, F.; PÊGO-FERNANDES, P. M.; FIORELLI, A. I.; ABENSUR, H.; MENEGHETTI, J. C.; GUTIERREZ, P. S.; VERGINELLI, G.; JATENE, A. D. - Evolução clínica e comportamento da função ventricular no pós-operatório tardio da cardiomioplastia. Rev. Bras. Cir. Cardiovasc., 10 (1): 3-17, 1995.

Além dos efeitos hemodinâmicos já comentados, é importante destacar que a melhora do desempenho sistólico do coração com a cardiomioplastia ocorre através da ação de um tecido externo ao miocárdio. Não se eleva, portanto, o consumo de oxigênio pelo miocárdio, uma vez que o trabalho ventricular é parcialmente transferido para o músculo auxiliar ${ }^{23}$. Esse fato, associado à queda da tensão sobre a parede ventricular, pode ser responsável pela recuperação parcial da contratilidade do miocárdio e pela interrupção da progressão da cardiopatia de base após a cardiomioplastia ${ }^{26}$. A esse respeito, a relação pressão-volume na câmara ventricular esquerda foi estudada em 8 pacientes desta série após o primeiro ano de pós-operatório, com o estimulador ligado e desligado por 24 horas ${ }^{2}$. Reduções significativas do estresse sistólico do ventrículo esquerdo de $175 \pm 12$ para $149 \pm 10 \mathrm{~g} / \mathrm{cm}^{2}$ $(p<0,05)$ e do estresse diastólico de $69 \pm 8$ para $37 \pm 5$ $\mathrm{g} / \mathrm{cm} 2(\mathrm{p}<0,01)$ foram demonstradas nesse estudo, quando as medidas obtidas com o estimulador ligado foram comparadas com as realizadas com o aparelho desligado. A queda significativa da rigidez da câmara ventricular esquerda e a melhora de sua elastância máxima foram também documentadas na presença da contração síncrona do enxerto muscular.

Em relação ao comportamento da função ventricular no pós-operatório tardio da cardiomioplastia, nesta investigação foi observada a manutenção da melhora dos parâmetros hemodinâmicos, demonstrada logo após o período de adaptação do enxerto muscular, até o quinto ano de seguimento. Esse fato poderia indicar a estabilização do processo de progressão da cardiopatia de base nos pacientes que sobreviveram tardiamente. Entretanto, também foi observada a tendência a queda da fração de ejeção e do encurtamento segmentar do VE a partir do segundo ano de seguimento, sugerindo que os efeitos da cardiomioplastia sobre a fisiopatologia da insuficiência cardíaca podem estar limitados a poucos anos de pós-operatório. A esse respeito, a análise da evolução clínica dos 5 pacientes que completaram 5 anos de seguimento mostra a progressão dos sintomas de insuficiência cardíaca após esse período e uma elevada mortalidade em 6 anos.

Não existem publicações prévias que mostrem a diminuição dos benefícios observados com a cardiomioplastia no pós-operatório tardio do procedimento. As experiências relatadas são limitadas, no entanto, a períodos mais curtos de seguimento e pela existência de afecções de diversas etiologias $8,10,19$. A progressão da cardiopatia de base e a diminuição da eficiência da cardiomioplastia a longo prazo são as explicações mais prováveis para os resultados tardios observados neste estudo. A esse respeito, alterações morfológicas do enxerto muscular foram observadas em 9 pacientes submetidos a avaliação anatomopatológica nesta investigação, tendo sido também previamente documentadas em 5 pacientes desta série através da ressonância magnética, em estudos realizados entre 26 meses e 55 meses de pós-operatório ${ }^{21}$.

\section{Alteraçōes do Rítmo Cardíaco}

A influência da cardiomioplastia sobre a incidência de arritmias em pacientes portadores de insuficiência miocárdica não tem sido discutida na literatura. Os resultados desta investigação mostram que o procedimento não modificou a incidência de fibrilação atrial e de arritmias ventriculares em pacientes portadores de cardiomiopatia dilatada durante o período estudado, apesar da melhora da condição clínica e da função ventricular esquerda. As arritmias ventriculares são freqũentes em pacientes com cardiomiopatia dilatada, mas a sua gravidade não tem sido relacionada de modo consistente com o grau de comprometimento da função ventricular ${ }^{17}$, situação que pode explicar os achados deste estudo. Com relação à fibrilação atrial, não existem evidências de que essa arritmia seja fator determinante de prognóstico em pacientes com insuficiência miocárdica ${ }^{11}$; além disso, foi controlada, em nossos pacientes, com o uso regular de amiodarona, não sendo responsável por qualquer complicação clínica durante o período de seguimento analisado.

\section{Evolução Clínica e Sobrevida em 5 Anos}

A melhora significativa da classe funcional dos pacientes no pós-operatório da cardiomioplastia tem sido demonstrada por vários autores $8,12,16,27,30$, sendo também observada nesta investigação. Além da melhora funcional, o estudo sobre a análise da qualidade de vida em pacientes da série demonstrou a diminuição das limitações apresentadas em diversos segmentos da vida diária, como na atividade física, nos padrões de sono e alimentação e no desempenho profissional ${ }^{7}$. A melhora da capacidade física após a cardiomioplastia também foi documentada por nós e por outros autores através da avaliação do desempenho cardiopulmonar durante o teste de esforço em esteira ergométrica ${ }^{10,34}$.

Por outro lado, apesar dos benefícios documentados no pós-operatório da cardiomioplastia, neste estudo, a sobrevida tardia foi de apenas $69,7 \%$ em 2 anos e de $39,6 \%$ aos 5 anos de seguimento. Esses resultados foram semelhantes aos relatados em estudos multicêntricos internacionais ${ }^{15,16}$ e em outras publicações que incluem pacientes com 
MOREIRA, L. F. P.; STOLF, N. A. G.; BOCCHI, E. A.; BACAL, F.; PÊGO-FERNANDES, P. M.; FIORELLI, A. I.; ABENSUR, H.; MENEGHETTI, J. C.; GUTIERREZ, P. S.; VERGINELLI, G.; JATENE, A. D. - Evolução clínica e comportamento da função ventricular no pós-operatório tardio da cardiomioplastia. Rev. Bras. Cir. Cardiovasc., 10 (1): 3-17, 1995.

miocardiopatia de várias etiologias $10,14,19$. Apenas BRAILE et alii 8 relataram sobrevida de mais de $80 \%$ em 5 anos para pacientes portadores de cardiomiopatia dilatada. Os pacientes operados, entretanto, tinham um comprometimento moderado da função ventricular esquerda pela ecocardiografia e não apresentavam sinais de hipertensão pulmonar no pré-operatório.

As causas de óbito no seguimento tardio após a cardiomioplastia têm sido geralmente relacionadas à progressão da insuficiência cardíaca ou à morte súbita. A progressão da insuficiência cardíaca tem ocorrido principalmente em pacientes que não apresentam melhora significativa da função ventricular após a operação. Esse fato pode estar relacionado a fatores pré-operatórios, como o grau de comprometimento funcional dos pacientes 10,30 ou a fatores relativos ao próprio procedimento cirúrgico, como a ocorrência de lesão tecidual do enxerto muscu$\operatorname{lar}^{3} \mathrm{e}$ o grau de envolvimento das câmaras ventriculares pelo músculo esquelético ${ }^{13}$. Outras causas de progressão da insuficiência cardíaca estão relacionadas, no entanto, à própria cardiopatia de base, como a ocorrência de miocardite aguda, infarto do miocárdio ou tromboembolismo pulmonar 8,30 .

Apesar da incidência de morte súbita nesta e em outras séries relatadas na literatura 8,10 ter sido menor do que a descrita em pacientes com insuficiência cardíaca em fase avançada, de aproximadamente $20 \%$ a $30 \%$ por ano 25 , a sua prevenção representa um grande desafio no seguimento de pacientes submetidos à cardiomioplastia. Do ponto de vista fisiopatológico, a morte súbita pode ser vista como a interação entre anormalidades estruturais do coração, distúrbios funcionais transitórios e eventos eletrofisiológicos específicos responsáveis pelas arritmias fatais. No entanto, a despeito dos efeitos da operação sobre a função sistólica e diastólica do ventrículo esquerdo e sobre o consumo de oxigênio pelo miocárdio, o valor relativo de cada fator envolvido com a morte súbita ainda é controverso, permanecendo imprecisa a influência do procedimento sobre a sua incidência.

Com relação à sobrevida no pós-operatório tardio da cardiomioplastia, é importante ressaltar que a operação foi indicada principalmente em pacientes com elevado risco de mortalidade em um ano, uma vez que os critérios de indicação não diferiram daqueles empregados no transplante cardíaco ${ }^{40}$. A esse respeito, um estudo comparativo não randomizado, realizado em pacientes portadores de cardiomiopatia dilatada ou de miocardiopatia da doença de Chagas, mostrou que a sobrevida dos pacientes submetidos à cardiomioplastia foi de $78,7 \%$ no primeiro ano de pós-operatório e de $65,1 \%$ no segundo ano, enquanto que os pacientes mantidos em tratamento clínico apresentaram uma sobrevida de apenas $41,1 \%$ em 1 ano e de $27,4 \%, 2$ anos após a indicação do procedimento 32,34 .

Também importante é a observação de que o risco de mortalidade até os 5 anos de seguimento foi maior em pacientes operados em classe funcional IV do que nos operados em classe III, sendo também sugerida a influência da resistência vascular pulmonar no pré-operatório sobre os resultados da cardiomioplastia. A importância desses fatores havia sido previamente identificada por outros autores $10,14,16$, mas os estudos relatados incluíam casos em que a cardiomioplastia foi associada a outros procedimentos. Embora essas publicações tenham mencionando a influência de outros fatores sobre a sobrevida no pós-operatório tardio, nesta série, não foi observada qualquer relação da fração de ejeção das câmaras ventriculares e das arritmias no pré-operatório com os resultados relatados.

\section{Aspectos da Anatomopatologia}

Apesar de alguns autores não terem descrito alterações degenerativas nos músculos esqueléticos utilizados experimentalmente na cardiomioplastia ${ }^{22}$, outros estudos têm relatado a ocorrência dessas alterações algumas semanas após o procedimento ${ }^{24} \mathrm{e}$ com a estimulação crônica do enxerto muscular ${ }^{28}$.

Nesta investigação, o estudo anatomopatológico mostrou a existência de intensa fibrose perimisial e atrofia importante das fibras musculares em paciente que faleceu aos 10 meses de seguimento e havia apresentado elevação importante da enzima creatinoquinase no pós-operatório imediato. Além disso, sinais de degeneração gordurosa foram observados em 9 estudos realizados em pacientes que faleceram com mais de 20 meses de seguimento, sendo de grau mais intenso nos 4 pacientes que faleceram após os 5 anos de pós-operatório.

A existência de fibrose e atrofia nos enxertos musculares tem sido descrita em estudos experimentais e é associada à ocorrência de lesão tecidual durante o procedimento ${ }^{24}$. $\mathrm{Na}$ experiência clínica, esse fato é relatado por alguns autores 12,30 , tendo sido sugerida a importância da dosagem plasmática da enzima creatinoquinase para o seu diagnóstico ${ }^{30}$.

Com relação à infiltração gordurosa, estudos experimentais mostram que essa alteração pode ocorrer após alguns meses de estimulação contínua do enxerto muscular ${ }^{28}$. A lesão do nervo toracodorsal pela estimulação elétrica persistente e $a$ isquemia relativa do enxerto provocada pela sua contração por períodos prolongados são as causas mais prováveis dessa degeneração, cuja instalação parece ser progressiva. A esse respeito, a existência de 
MOREIRA, L. F. P.; STOLF, N. A. G.; BOCCHI, E. A.; BACAL, F.; PÊGO-FERNANDES, P. M.; FIORELLI, A. I.; ABENSUR, H.; MENEGHETTI, J. C.; GUTIERREZ, P. S.; VERGINELLI, G.; JATENE, A. D. - Evolução clínica e comportamento da funçăo ventricular no pós-operatório tardio da cardiomioplastia. Rev. Bras. Cir. Cardiovasc., 10 (1): 3-17, 1995.

infiltração gordurosa foi observada nesta investigação apenas nos estudos anatomopatológicos dos pacientes com mais de 20 meses de seguimento, sendo significativamente relacionada com o tempo. A existência e o grau das alterações morfológicas do enxerto muscular, compatíveis com diminuição da densidade das fibras musculares e com infiltração gordurosa, em estudos realizados entre 26 e 55 meses após a cardiomioplastia através da ressonância magnética nos 4 pacientes que faleceram após os 5 anos de seguimento ${ }^{21}$, também corroboram com o caráter progressivo do comprometimento.

Outros autores relataram a ausência de alterações morfológicas significativas em enxertos musculares de pacientes submetidos a necropsia ou transplante cardiaco no pós-operatório tardio da cardiomioplastia ${ }^{10,19}$. No entanto, a estimulação do músculo grande dorsal nesses pacientes foi sincronizada a cada dois batimentos ventriculares durante a maior parte do seguimento pós-operatório, enquanto que os enxertos musculares dos nossos pacientes foram cronicamente estimulados em sincronia a todos os batimentos cardíacos.

\section{Consideraçōes Finais}

Nesta investigação e em outras experiências relatadas na literatura, existem evidências significativas da influência positiva da cardiomioplastia sobre a evolução clínica e o comportamento da função ventricular em pacientes com insuficiência miocárdica em fase avançada. Por outro lado, os resultados da operação são limitados pela elevada mortalidade tardia apresentada por pacientes operados em classe funcional IV, sugerindo que ela deva ser indicada apenas em fases mais precoces de comprometimento da função ventricular. Nesse sentido, a cardiomioplastia pode ser realizada com baixo risco de mortalidade em pacientes com cardiomiopatia dilatada e não impede a posterior realização do transplante cardíaco nos casos de progressão da doença de base ${ }^{20}$. Além disso, neste estudo e em outros relatos da literatura $8,10,16$, os pacientes operados em classe funcional III têm apresentado uma sobrevida em 5 anos semelhante à do transplante cardíaco ${ }^{38}$ e superior aos resultados da terapêutica medicamentosa. Publicações recentes, a respeito do tratamento da insuficiência cardíaca com o uso de inibidores da enzima de conversão e de vasodilatadores arteriolares, mostram uma sobrevida de apenas $40 \%$ em 5 anos para pacientes em classe funcional III. Outros estudos relatam uma expectativa de vida ainda mais restrita em pacientes com alterações persistentes do comportamento hemodinâmico, situação que foi observada em todos os pacientes desta série no pré-operatório ${ }^{35}$.

Finalmente, apesar das perspectivas da cardiomioplastia no tratamento das miocardiopatias, alguns aspectos de sua utilização ainda devem ser objeto de estudos clínicos ou experimentais, antes que essa operação seja definitivamente incorporada na terapêutica cirúrgica da insuficiência miocárdica. Existem controvérsias a respeito da técnica cirúrgica empregada e dos protocolos utilizados na estimulação muscular esquelética para a obtenção de maior eficiência do procedimento. Paralelamente, a isquemia do enxerto muscular no pós-operatório imediato e a possibilidade de degeneração gordurosa desse enxerto a longo prazo são complicaçōes cuja prevenção é fundamental para os resultados tardios da cardiomioplastia. 
MOREIRA, L. F. P.; STOLF, N. A. G.; BOCCHI, E. A.; BACAL, F.; PÊGO-FERNANDES, P. M.; FIORELLI, A. I.; ABENSUR, H.; MENEGHETTI, J. C.; GUTIERREZ, P. S.; VERGINELLI, G.; JATENE, A. D. - Evolução clínica e comportamento da função ventricular no pós-operatório tardio da cardiomioplastia. Rev. Bras. Cir. Cardiovasc., 10 (1): 3-17, 1995.

RBCCV 44205-253

MOREIRA, L. F.P.; STOLF, N. A. G.; BOCCHI, E. A.; BACAL, F.; PÊGO-FERNANDES, P. M.; FIORELLI, A. I.; ABENSUR, H.; MENEGHETTI, J. C.; GUTIERREZ, R. S.; VERGINELLI, G.; JATENE, A. D. - Clinical and ventricular function in the late follow-up of dynamic cardiomyoplasty. Rev. Bras. Cir. Cardiovasc., $10(1): 3-17,1995$.

ABSTRACT: Dynamic cardiomyoplasty has been proposed as an alternative to heart transplantation in the treatment of severe cardiomyopathies. In this investigation, its results were investigated in 34 patients with idiopathic dilated cardiomyopathy submitted to this procedure from May 1988 to September 1994. Twenty seven patients were in New York Heart Association functional class III and 7 were in class IV before the operation, despite the use of maximal medical therapy. Hospital mortality was $2.9 \%$ and 1 patient presenting heart failure progression was submitted to urgent heart transplantation 42 days after cardiomyoplasty. The other patients were followed-up from 2 to 73 months (mean, 27.4 months). At 6 months of follow-up, 12 patients were in functional class $\mathrm{I}, 15$ patients in class II and 3 patients in class III ( $p=0.001$, in relation to preoperative data). Similar findings were observed from one to four years of follow-up. On the other hand, 14 patients died up to 5 years after the operation and the survival rates were $84.7 \%$ at 1 year, $67.7 \%$ at 2 years and $39.9 \%$ at 5 years of follow-up. The cause of death was progression of heart failure in 9 patients and 5 patients died suddenly. By stepwise Cox regression method, patients operated in functional class IV had relative risk of death 5.5 times higher than class III patients $(p=0.006)$, whose survival rate was $52.7 \%$ at 5 years of followup. Regarding cardiomyoplasty influence on ventricular function, radioisotopic left ventricular (LV) ejection fraction improved from $19.8+3.1 \%$ to $23.6+6.2 \%$, at 6 months of follow-up. Doppler-echocardiography documented similar changes in LV wall shortening. Also, right heart catheterization showed significant increases in mean arterial pressure, stroke index and LV stroke work index, which were associated with the decrease of mean pulmonary wedge pressure. On the other hand, it was not found any significant postoperative change in ventricular arrhythmia and atrial fibrillation incidence. At the late postoperative period, the hemodynamic improvement was maintained up to 5 years of follow-up, while LV ejection fraction and wall shortening tended to decrease after the second postoperative year returned to the preoperative levels. Finally, 3 of the 5 patients who completed 5 years of follow-up died due to heart failure progression and other died suddenly up to 73 months after the operation. Furthermore, histological examinations showed important fat tissue infiltration in the skeletal muscle flap of 9 patients submitted to necropsy studies from 20 to 73 months of follow-up. These histological changes were more severe in patients with longer follow-up. In conclusion, dynamic cardiomyoplasty improves congestive heart failure and LV function in patients with idiopathic dilated cardiomyopathy. However, long-term survival after this procedure is limited in patients operated upon in functional class IV. Furthermore, the cardiomyoplasty benefits on left ventricular function appear to be limited to a midterm period and this fact may be associated with late muscle flap degeneration.

DESCRIPTORS: Cardiomyoplasty. Heart failure, cardiomyopathy, surgery. Cardiomyopathies, surgery.

\section{REFERÊNCIAS BIBLIOGRÁFICAS}

1 AULER JÚNIOR, J. O. C.; MOREIRA, L. F. P.; CARVALHO, M. J.; STOLF, N. A. G.; AMARAL, R. V. G.; JATENE, A. D. - Anesthetic management of patients undergoing cardiomyoplasty. Anesthesiology, 77: $379-381,1992$.

2 BELLOTTI, G.; MORAES, A. V.; BOCCHI, E. A.; ARIE, S.; MEDEIROS, C.; MOREIRA, L. F. P.; JATENE, A. D.; PILEGGI, F. - Late effects of cardiomyoplasty on left ventricular mechanics and diastolic filling. Circulation, 88 (Supl. 2): 304-308, 1993.

3 BOCCHI, E. A.; BELLOTTI, G.; MOREIRA, L. F. P.; GUTIERREZ, P. S.; STOLF, N. A. G.; JATENE, A. D.; PILEGGI, F. - Prognostic indicators of one-year outcome after cardiomyoplasty for dilated cardiomyopathy. Am. J. Cardiol., 73: 604-608, 1994.
4 BOCCHI, E. A.; LOPES, H. F.; SILVA, H. B.; MORAES, A. V.; BACAL, F.; MOREIRA, L. F. P.; BELLOTTI, G.; STOLF, N. A. G.; JATENE, A. D.; PILEGGI, F. - Neuro-hormonal activity and baroreflex sensitivity after cardiomyoplasty. Eur. Heart. J., 15 (Abstract Suppl.) 274, 1994.

5 BOCCHI, E. A.; MOREIRA, L. F. P.; BELLOTTI, G.; BARRETO, A. C. P.; SERRO AZUL, L. G.; STOLF, N. A. G.; JATENE, A. D.; PILEGGI, F. - Hemodynamic study during upright isotonic exercise before and six months after dynamic cardiomyoplasty for idiopathic dilated cardiomyopathy or Chagas' disease. $A m$. J. Cardiol., 67: 213-214, 1991.

6 BOCCHI, E. A.; MOREIRA, L. F. P.; MORAES, A. V.; BELLOTTI, G.; GAMA, M.; STOLF, N. A. G.; JATENE, A. D.; PILEGGI, F. - Effects of dynamic 
MOREIRA, L. F. P.; STOLF, N. A. G.; BOCCHI, E. A.; BACAL, F.; PÊGO-FERNANDES, P. M.; FIORELLI, A. I.; ABENSUR, H.; MENEGHETTI, J. C.; GUTIERREZ, P. S.; VERGINELLI, G.; JATENE, A. D. - Evolução clínica e comportamento da função ventricular no pós-operatório tardio da cardiomioplastia. Rev. Bras. Cir. Cardiovasc., 10 (1): 3-17, 1995.

cardiomyoplasty on regional wall motion, ejection fraction and geometry of left ventricle. Circulation, 86 (Supl. 2): 231-235, 1992.

BORGHETTI-MAIO, S. A.; ROMANO, B. W.; BOCCHI, E. A.; MOREIRA, L. F. P.; BARRETO, A. C. P.; STOLF, N. A. G.; BELLOTTI, G.; PILEGGI, F.; JATENE, A. D. - Quality of life after cardiomyoplasty. J. Heart Lung Transplant., 13: 271-275, 1994.

BRAILE, D. M.; SOARES, M. J. F.; RODRIGUES, M. C. Z.; RAMIN, S. L.; THEVENARD, R. S.; ZAIANTCHICK, M.; SOUZA, D. R. S. Cardiomioplastia: estudo clínico de 26 pacientes em 6 anos. Rev. Bras. Marcapasso Arritmia, 6: 71-83, 1993.

CARPENTIER, A. \& CHACHQUES, J. C. - The use of stimulated skeletal muscle to replace diseased human heart muscle. In: CHIU, R. C. - J. (ed.) Biomechanical cardiac assist: cardiomyoplasty and muscle-powered devices. Mount Kisco, New York, Futura, 1986. p. 85-101.

CARPENTIER, A.; CHACHQUES, J. C.; ACAR, C.; RELLAND, J.; MIHAILEANU, S.; BENSASSON, D.; KIEFFER, J. P.; GUIBOURT, P.; TOURNAY, D.; ROUSSIN, J.; GRANDJEAN, P. A. - Dynamic cardiomyoplasty at seven years. J. Thorac. Cardiovasc. Surg., 106: 42-53, 1993.

11 CARSON, P. E.; JOHNSON, G. R.; DUNKMAM, B.; FLETCHER, R. D.; FARREL, L.; COHN, J. N. - The influence of atrial fibrillation on prognosis in mild to moderate heart failure. Circulation, 87 (Supl. 4): 102 $110,1993$.

CHACHQUES, J. C.; ACAR, C.; PORTOGHESE, M.; BENSASSON, D.; GUIBOURT, P.; GRARE, P. H.; JEBARA, V. A.; GRANDJEAN, P. A.; CARPENTIER, A. - Dynamic cardiomyoplasty for long-term cardiac assist. Eur. J. Cardio-Thorac. Surg., 6: 642-648, 1992.

CHIU, R. C. J.; ODIM, J. N. K.; BURGESS, J. H.; (THE McGILL CARDIOMYOPLASTY GROUP) - Responses to dynamic cardiomyoplasty for idiopathic dilated cardiomyopathy. Am. J. Cardiol., 72: 475-479, 1993.

FURNARY, A. P.; MAGOVERN, J. A.; CHRISTLIEB, I. Y.; ORIE, J. E.; SIMPSON, K. A.; MAGOVERN, G. J. - Clinical cardiomyoplasty: preoperative factors associated with outcome. Ann. Thorac. Surg., 54: 1139-1143, 1992.

FURNARY, A. P.; MOREIRA, L. F. P.; JESSUP, M.; (THE AMERICAN CARDIOMYOPLASTY GROUP) Dynamic cardiomyoplasty improves systolic ventricular function. Circulation, 90 (Supl 1): 309, 1994. TERPSTRA, B.; BOURGEOIS, I. - Dynamic cardiomyoplasty: clinical follow-up results. J. Cardiac Surg., 6 (Supl.): 80-88, 1991.
JATENE, A. D.; MOREIRA, L. F. P.; STOLF, N. A. G.; BOCCHI, E. A.; SEFERIAN Jr., P.; PÊGO-FERNANDES, P. M.; ABENSUR, H. - Left ventricular function changes after cardiomyoplasty in patients with dilated cardiomyopathy. J. Thorac. Cardiovasc. Surg., 102: 132-139, 1991.

JEGADEN, O.; DELAHAYE, F.; FINET, G.; VAN DER VEEN, F. H.; MONTAGNA, P.; EKER, A.; OSSETE, J.; ROSSI, R.; SAINT-PIERRE, A.; MIKAELOFF, P. - Late hemodynamic results after cardiomyoplasty in congestive heart failure. Ann. Thorac. Surg., 57: 1151-1157, 1994.

JEGADEN, O.; DELAHAYE, F.; MONTAGNA, P.; VEDRINNE, C.; BLANC, P.; ROSSI, R.; TABIB, A.; SAINT-PIERRE, A.; DELAHAYE, J. P.; MIKAELOFF, P. H. - Cardiomyoplasty does not preclude heart transplantation. Ann. Thorac. Surg., 53: 875-881, 1992.

KALIL FILHO, R.; BOCCHI, E. A.; WEISS, R. G.; ROSEMBERG, L.; BACAL, F.; MOREIRA, L. F. P.; STOLF, N. A. G.; BELLOTTI G.; JATENE, A. D.; PILEGGI, F. - Magnetic resonance imaging evaluation of chronic changes in the latissimus dorsi cardiomyoplasty. Circulation, 90 (Supl. 2): 102-106, 1994.

KAO, R. L.; CHRISTLIEB, I. Y.; MAGOVERN, G. J.; PARK, S. B.; MAGOVERN Jr., G. J. - The importance of skeletal muscle fiber orientation for dynamic cardiomyoplasty. J. Thorac. Cardiovasc. Surg., 99: 134-140, 1990.

KAWAGUCHI, O.; GOTO, Y.; FUTAKI, S.; OHGOSHI, Y.; YAKU, H.; SUGA, H. - Mechanical enhancement and myocardial oxygen saving by synchronized dynamic left ventricular compression. J. Thorac. Cardiovasc. Surg., 103: 573-581, 1992.

KRATZ, J. M.; JOHNSON, W. S.; MUKHERJEE, R.; HU, J.; CRAWFORD, F. A.; SPINALE, F. G. - The relation between latissimus dorsi skeletal muscle structure and contractile function after cardiomyoplasty. J. Thorac. Cardiovasc. Surg., 107: 868-878, 1994.

LARSEN, L.; MARKHAM, J.; HAFFAJEE, C. I. - Sudden death in idiopathic dilated cardiomyopathy: role of ventricular arrhythmias. PACE, 16: 1051-1059, 1993.

LEE, K. F.; DIGNAN, R. J.; PARMAR, J. M.; DYKE, C. M.; BENTON, G.; YEH Jr., T.; ABD-ELFATTAH, A. S.; WECHSLER, A. S. - Effects of dynamic cardiomyoplasty on left ventricular performance and myocardial mechanics in dilated cardiomyopathy. J. Thorac. Cardiovasc. Surg., 102: 124-131, 1991. 
MOREIRA, L. F. P.; STOLF, N. A. G.; BOCCHI, E. A.; BACAL, F.; PEGGO-FERNANDES, P. M.; FIORELLI, A. I.; ABENSUR, H.; MENEGHETTI, J. C.; GUTIERREZ, P. S.; VERGINELLI, G.; JATENE, A. D. - Evolução clínica e comportamento da função ventricular no pós-operatório tardio da cardiomioplastia. Rev. Bras. Cir. Cardiovasc., 10 (1): 3-17, 1995.

LORUSSO, R.; ZOGNO, M.; LA CANNA, G.; METRA, M.; SANDRELLI, L.; BORGHETTI, V.; MAISANO, F.; ALFIERI, O. - Dynamic cardiomyoplasty as an effective therapy for dilated cardiomyopathy. $J$. Cardiac Surg., 8: 177-183, 1993.

LUCAS, C. M. H. B.; VAN DER VEEN, F. H.; CHERIEX, E. C.; LORUSSO, R.; HAVENITH, M.; PENN, O. C. K. M.; WELLENS, H. J. J. - Long-term follow-up (1235 weeks) after dynamic cardiomyoplasty. J. Am. Coll. Cardiol., 22: 758-767, 1993.

MAGOVERN, J. A.; PARK, S. E.; CMOLIK, B. L.; TRUMBLE, D. R.; CHRISTLIEB, I. Y.; MAGOVERN, G. J. - Early effects of right latissimus dorsi cardiomyoplasty on left ventricular function. Circulation, 88 (Supl. 2) 298-303, 1993.

30 MOREIRA, L. F. P.; BOCCHI, E. A.; STOLF, N. A. G.; PILEGGI, F.; JATENE, A. D. - Current expectations in dynamic cardiomyoplasty. Ann. Thorac. Surg., 55: 299-303, 1993.

31 MOREIRA, L. F. P.; CHAGAS, A. C. P.; CAMARANO, G. P.; LEIRNER, A.; PÊGO-FERNANDES, P. M.; LUZ, P. L.; STOLF, N. A. G.; JATENE, A. D. Cardiomyoplasty benefits in experimental myocardial dysfunction. J. Cardiac Surg., 4: 164-170, 1989.

MOREIRA, L. F. P.; SEFERIAN JÚNIOR, P.; BOCCHI, E. A.; PÊGO-FERNANDES, P. M.; STOLF, N. A. G.; BARRETTO, A. C. P.; JATENE, A. D. - Survival improvement with dynamic cardiomyoplasty in patients with dilated cardiomyopathy. Circulation, 84 (Supl. 3) 296-302, 1991.

33 MOREIRA, L. F. P.; STOLF, N. A. G.; BOCCHI, E. A.; BARRETO, A. C. P.; MENEGHETTI, J. C.; GIORGI, M. C. P.; MORAES, A. V.; LEITE, J. J.; LUZ, P. L.; JATENE, A. D. - Latissimus dorsi cardimyoplasty in the treatment of patients with dilated cardiomyopathy. Circulation, 82 (Supl. 4): 257-263, 1990.
MOREIRA, L. F. P.; STOLF, N. A. G.; BOCCHI, E. A.; SEFERIAN JÚNIOR, P.; PÊGO-FERNANDES, P. M.; BARRETO, A. C. P.; ABENSUR, H.; MENEGHETTI, J. C.; JATENE, A. D. - Resultados da cardiomioplastia no tratamento da cardiomiopatia dilatada. Rev. Bras. Cir. Cardiovasc., 6: 85-95, 1991.

MORLEY, D. \& BROZENA, S. C. - Assessing risk by hemodynamic profile in patients awaiting cardiac transplantation. Am. J. Cardiol., 73: 379-383, 1994.

PACKER, M. - Pathophysiology of chronic heart failure. Lancet, 340: 88-92, 1992.

37 PITT, B.; COHN, J. N.; FRANCIS, G. S.; KOSTIS, J. B.; PARKER, M.; PFEFFER, M. A.; SWEDBERG, K.; YUSUF, S. - The effect of treatment on survival in congestive heart failure. Clin. Cardiol., 15: 323-329, 1992.

SARRIS, G. E.; MOORE, K. A.; SCHROEDER, J. S.; HUNT, S. A.; FOWLER, M. B.; VALANTINE, H. B.; VAGELOS, R. H.; BILLINGHAM, M. E.; OYER, P. E.; STINSON, E. B.; REITZ, B. A.; SHUMWAY, N. E. Cardiac transplantation: the Stanford experience in the cyclosporine era. J. Thorac. Cardiovasc. Surg., 108: 240-252, 1994.

39 STEVENSON, L. W.; WARNER, S. L.; STEIMLE, A. E.; FONAROW, G. C.; HAMILTON, M. A.; MORIGUSHI, J. D.; KOBASHIGAWA, J. A.; TILLISCH, J. H.; DRINKWATER, D. C.; LAKS, H. - The impending crisis awaiting cardiac transplantation: modeling a solution based on selection. Circulation, 89: 450457, 1994.

40 VAGELOS, R. \& FOWLER, M. B. - Selection of patients for cardiac transplantation. Cardiol. Clin., 8: 23-38, 1990. 